\title{
Electric dipole moments and dark matter in a $C P$ violating MSSM
}

\author{
Tomohiro Abe, ${ }^{1,2}$ Naoya Omoto, ${ }^{3}$ Osamu Seto, ${ }^{4,3}$ and Tetsuo Shindou ${ }^{5}$ \\ ${ }^{1}$ Institute for Advanced Research, Nagoya University, Furo-cho Chikusa-ku, \\ Nagoya, Aichi 464-8602, Japan \\ ${ }^{2}$ Kobayashi-Maskawa Institute for the Origin of Particles and the Universe, \\ Nagoya University, Furo-cho Chikusa-ku, Nagoya, Aichi 464-8602 Japan \\ ${ }^{3}$ Department of Physics, Hokkaido University, Sapporo 060-0810, Japan \\ ${ }^{4}$ Institute for International Collaboration, Hokkaido University, Sapporo 060-0815, Japan \\ ${ }^{5}$ Division of Liberal-Arts, Kogakuin University, Nakano-machi, Hachioji, Tokyo 192-0045, Japan
}

(Received 24 May 2018; published 30 October 2018)

\begin{abstract}
We investigate electric dipole moments (EDMs) in a $C P$-violating minimal supersymmetric standard model with the binolike neutralino dark matter (DM) annihilating through the heavy Higgs funnel. Motivated by the current experimental results, in particular, the measured mass of the standard model-like Higgs boson, we consider a mass spectrum with stop masses of about $10 \mathrm{TeV}$. For the other sfermions, we consider masses of about $100 \mathrm{TeV}$. We show that $C P$-violating phases of the order of ten degrees in gaugino and Higgsino mass parameters are consistent with the current bound by EDMs of the electron, the neutron, and the mercury. They are within the reach of future experiments. We also show that effects of $C P$-violating phases induce a difference in DM-nucleon scattering cross section by a factor.
\end{abstract}

DOI: 10.1103/PhysRevD.98.075029

\section{INTRODUCTION}

Supersymmetry is an attractive candidate of physics beyond the standard model (SM), although current results from LHC experiments indicate that supersymmetric (SUSY) particles are heavier than have been expected. Attractive aspects come from the fact that, for instance, the gauge coupling unification is realized in the minimal SUSY SM (MSSM), the gauge hierarchy problem is improved, and elementary scalar fields such as Higgs fields are introduced in a theoretically natural way. Moreover, SUSY models may provide additional interesting consequences. SUSY interpretation of muon anomalous magnetic moment is one example [1-3]. SUSY models contain new sources of $C P$ violation and/or flavor violation, which potentially induce new $C P$ or flavor violating phenomena. The lightest SUSY particle (LSP) is stable and hence a good candidate for dark matter (DM) in our Universe, if the R-parity is unbroken [4,5].

Electric dipole moments (EDMs) of the neutron and other heavy atoms are prime physical quantities for probing sources of $C P$ violation. Parameters in the MSSM generally pose several $C P$ violating phases. It used to be regarded that the null experimental EDM results confront the MSSM with

Published by the American Physical Society under the terms of the Creative Commons Attribution 4.0 International license. Further distribution of this work must maintain attribution to the author(s) and the published article's title, journal citation, and DOI. Funded by SCOAP .
$\mathcal{O}$ (1) $C P$ violating phases and $\mathcal{O}(100) \mathrm{GeV}$ masses of SUSY particles [6-9]. The LHC results suggest that masses of many SUSY particles are larger than $\mathcal{O}(10) \mathrm{TeV} .{ }^{1}$ Therefore $C P$ violating phases of order unity in the SUSY sector $[10,11]$ seems still likely and worth investigating. For recent studies, see e.g., Refs. [12,13].

In the MSSM with R-parity, the lightest neutralino $\tilde{\chi}$ is a candidate of the weakly interacting mass particle (WIMP) DM. While LHC experiments as well as direct detection experiments of DM, such as LUX [14,15], XENON1T [16], and PandaX-II $[17,18]$ are constraining large parameter space of the MSSM, there are still viable scenarios reproducing thermal relic abundance of the DM consistently. Appropriate magnitude of annihilation cross section of neutralino in the early Universe is realized if (i) neutralinos annihilate significantly through $\mathrm{SU}(2)$ gauge interaction, or (ii) annihilation cross section of binolike neutralino is enhanced with a particular mass spectrum of other associated particles.

Higgsino-like neutralino DM with the mass of about $1 \mathrm{TeV}$ is an example in the former class. Phenomenology in this scenario such as the direct detection of DM, contribution to the EDMs, and collider signals have been precisely studied in Ref. [19].

In this paper, we focus on another case in the later class; a binolike neutralino DM annihilates through heavy Higgs

\footnotetext{
${ }^{1}$ To be precise, a scenario with SUSY particles with masses of a few $\mathrm{TeV}$ is still allowed. The current limit on gluino mass is around $2 \mathrm{TeV}$ and the squark masses can be smaller than $3 \mathrm{TeV}$ in the degenerate case.
} 
boson resonance [20-24]. ${ }^{2}$ In this scenario where heavy Higgs boson resonance in the neutralino DM annihilation is utilized, masses of the heavy Higgs boson are about twice of the mass of the neutralino DM. Since the binolike neutralino contains small Higgsino component, the neutralino can be searched through Higgs bosons exchange by spinindependent scattering off nucleus [26]. Masses of stops would be around $10 \mathrm{TeV}$ in order to reproduce the measured SM-like Higgs boson mass $\left(m_{h}=125 \mathrm{GeV}\right)[27,28]$. Then, all the other SUSY particle masses and parameters except for the bino mass, the Higgsino mass parameter $\mu, B \mu$, and stop masses can be much larger than $\mathcal{O}(1) \mathrm{TeV}$. With such SUSY particle mass spectrum, most of SUSY contributions to the low energy phenomena can be decoupled as the irrelevant SUSY particles are heavier, SUSY contributions to the EDMs in $C P$ violating models can still be significantly large nevertheless. The main goal of this article is, by decoupling the other particles, to estimate the magnitude of EDMs induced by $C P$ violation in neutralino DM sector with taking account of $C P$-violating phase effects into the thermal DM abundance [29-34].

We examine the electron EDM, the nucleon EDM, and the mercury EDM, as well as the DM-nucleon scattering cross section on the parameter space, where appropriate thermal DM relic abundance is reproduced, for order unity $C P$-violating phases of gaugino mass parameters and the $\mu$ parameter. For non-vanishing $C P$ phase of $\mu$ and $A$ parameters, see, e.g., Refs. [29,32,35,36]. The magnitude of scattering off cross section between DM and nucleon is affected by the $C P$ violating phases [32,37-42]. We also study the dependence of spin-independent cross section of the DM in our scenario and find that the effect changes by a factor. We show that wide parameter regions in our scenario are now unconstrained yet, but will be explored by future experiments.

This article is organized as follows. In Sec. II, we define a benchmark scenario for studying phenomenology in our DM scenario. In Sec. III, we show the results of our analysis on several EDM measurements and the spin-independent cross section. Summary and conclusion are presented in Sec. IV.

\section{SETUP OF THE SCENARIO}

In this section, we briefly review the MSSM Lagrangian, and we describe the parameter setup for our analysis. The superpotential and the soft SUSY breaking terms in the MSSM are given by Ref. [43]

$$
\begin{aligned}
W= & \epsilon_{a b}\left[\left(y_{e}\right)_{i j} H_{1}^{a} L_{i}^{b} \bar{E}_{j}+\left(y_{d}\right)_{i j} H_{1}^{a} Q_{i}^{b} \bar{D}_{j}\right. \\
& \left.+\left(y_{u}\right)_{i j} H_{2}^{a} Q_{i}^{b} \bar{U}_{j}-\mu H_{1}^{a} H_{2}^{b}\right],
\end{aligned}
$$

\footnotetext{
${ }^{2}$ For a study of $C P$ violation in stau coannihilation scenario, see, e.g., Ref. [25].
}

and

$$
\begin{aligned}
\mathcal{L}_{\text {soft }}= & -\frac{M_{1}}{2} \tilde{B} \tilde{B}-\frac{M_{2}}{2} \tilde{W}^{\alpha} \tilde{W}^{\alpha}-\frac{M_{3}}{2} \tilde{G}^{A} \tilde{G}^{A} \\
& -m_{H_{1}}^{2} H_{1 a}^{*} H_{1}^{a}+m_{H_{2}}^{2} H_{2 a}^{*} H_{2}^{a} \\
& -\tilde{q}_{i L a}^{*}\left(M_{\tilde{q}}^{2}\right)_{i j} \tilde{q}_{j L}^{a}-\tilde{\ell}_{i_{L} a}^{*}\left(M_{\tilde{\ell}}^{2}\right)_{i j} \tilde{\ell}_{j L}^{a} \\
& -\tilde{u}_{i R}\left(M_{\tilde{u}}^{2}\right)_{i j} \tilde{u}_{j R}^{*}-\tilde{d}_{i R}\left(M_{\tilde{d}}^{2}\right)_{i j} \tilde{d}_{j R}^{*}-\tilde{e}_{i R}\left(M_{\tilde{e}}^{2}\right)_{i j} \tilde{e}_{j R}^{*} \\
& -\epsilon_{a b}\left[\left(T_{e}\right)_{i j} H_{1}^{a} \tilde{\ell}_{i L}^{b} \tilde{e}_{j R}+\left(T_{d}\right)_{i j} H_{1}^{a} \tilde{q}_{i L}^{b} \tilde{d}_{j R}\right. \\
& \left.+\left(T_{u}\right)_{i j} H_{2}^{a} \tilde{q}_{i L}^{b} \tilde{u}_{j R}+m_{3}^{2} H_{1}^{a} H_{2}^{b}+\text { H.c. }\right],
\end{aligned}
$$

respectively. The convention of the epsilon tensor is $\epsilon_{12}=-\epsilon_{21}=1$. Here, we note that gaugino mass parameter for bino $M_{1}$, wino $M_{2}$, and gluino $M_{3}$ are in general complex. In the following, we focus on the Yukawa couplings of the third generation quarks and leptons, so that we use $y_{t}, y_{b}$, and $y_{\tau}$ for the Yukawa couplings of top, bottom, and tau, respectively. Neglecting the flavor mixing in the soft SUSY breaking terms, we take flavor diagonal soft scalar masses as $M_{\tilde{q}_{i}}^{2}=\left(M_{\tilde{q}}^{2}\right)_{i i}, \quad M_{\tilde{e}_{i}}^{2}=\left(M_{\tilde{\ell}}^{2}\right)_{i i}, \quad M_{\tilde{u}_{i}}^{2}=\left(M_{\tilde{u}}^{2}\right)_{i i}$, $M_{\tilde{d}_{i}}^{2}=\left(M_{\tilde{d}}^{2}\right)_{i i}$, and $M_{\tilde{e}_{i}}^{2}=\left(M_{\tilde{e}}^{2}\right)_{i i}$. For the trilinear couplings, $A$ parameters defined by $\left(T_{u}\right)_{33}=A_{\tau} y_{t},\left(T_{d}\right)_{33}=A_{\tau} y_{b}$, and $\left(T_{e}\right)_{33}=A_{\tau} y_{\tau}$ are used.

In the MSSM, the mass of the SM-like Higgs boson is expressed with some SUSY breaking parameters. In our analysis, we take $\tan \beta:=\left\langle H_{2}\right\rangle /\left\langle H_{1}\right\rangle=30$ and we fix the stop mass parameters as $M_{\tilde{q}_{3}}=7 \mathrm{TeV}, M_{\tilde{t}}:=M_{\tilde{u}_{3}}=7 \mathrm{TeV}$ and $A_{t}=10 \mathrm{TeV}$, then the measured SM-like Higgs boson mass $m_{h} \simeq 125 \mathrm{GeV}$ can easily reproduced $[27,28]$. The other SUSY particles are relevant to neither the mass of the SM-like Higgs boson nor the DM relic density. We may assume that those are much heavier than stop so that those are decoupled from low energy observables. We here take masses of the other sfermions as $100 \mathrm{TeV}$ and the Wino and gluino masses to be $10 \mathrm{TeV}$. In this article, we focus on the binolike DM with the Higgs funnel scenario, where heavy Higgs boson mass is close to twice the mass of the DM so that the binolike neutralino rapidly annihilate through the heavy Higgs bosons resonance and has left with the appropriate cosmic abundance for DM. Since masses of heavier neutral Higgs bosons, $m_{H}$ and $m_{A}$, are close to the charged Higgs boson mass $m_{H^{ \pm}}$in the MSSM, we fix $m_{H^{ \pm}}$to be twice of bino mass parameter $M_{1}$ to realize resonant annihilation by the heavy Higgs bosons. In addition, the $\tilde{\chi}-\tilde{\chi}$-Higgs boson coupling depends on non-vanishing Higgsino component in the neutralino. Thus, both the bino mass $\left|M_{1}\right|$ and the Higgsino mass $|\mu|$ should be of the order of TeV. We leave $M_{1}$ as a free parameter and solve $|\mu|$ from the measured dark matter energy density. We summarize the parameter set in our analysis as follows:

$$
\left|M_{2}\right|=\left|M_{3}\right|=10 \mathrm{TeV},
$$




$$
\begin{gathered}
M_{\tilde{q}_{1,2}}=M_{\tilde{u}_{1,2}}=M_{\tilde{d}_{1,2,3}}=M_{\tilde{\ell}_{1,2,3}}=M_{\tilde{e}_{1,2,3}}=100 \mathrm{TeV} \\
M_{\tilde{q}_{3}}=M_{\tilde{t}}=7 \mathrm{TeV} \\
A_{t}=10 \mathrm{TeV} \\
m_{H^{ \pm}}=2 M_{1} \\
\tan \beta=30
\end{gathered}
$$

The other $A$-terms are zero. With the above parameter set, besides the Cabibbo-Kobayashi-Maskawa (CKM) phase and the $C P$ phases in the sfermion mass matrices, five parameters, $\mu$, gaugino masses $M_{i}$ and $A_{t}$, may have $C P$ phases $\left(\phi_{\mu}, \phi_{M_{1}}, \phi_{M_{2}}, \phi_{M_{3}}, \phi_{A_{t}}\right)$, respectively. Here, each phases of a quantity $X$ are defined by $X=|X| e^{i \phi_{X}}$.

There is a rephasing degree of freedom in the MSSM. Thus, all the physical quantities are described by the following combinations,

$$
\begin{array}{lll}
\arg \left(M_{i} M_{j}^{*}\right), & \arg \left(M_{i} A_{t}^{*}\right), & \arg \left(\mu M_{i}\right), \\
\arg \left(\mu A_{t}\right), & (i, j=1,2,3) .
\end{array}
$$

Without loss of generality, we can take the basis of $C P$ phases as $\phi_{M_{3}}=0$. In addition, we take $\phi_{A_{t}}=0$ to concentrate on the $C P$ violation in the neutralino sector as well as, technically speaking, to keep $m_{h} \simeq 125 \mathrm{GeV}$ avoiding complicated parameter dependence of the SM-like Higgs boson mass. In general, the nonzero value of $\phi_{A_{t}}$ significantly contributes to the predictions of the EDMs. However, in our parameter set given in Eqs. (2.3)-(2.8), we find the contribution from $\phi_{A_{t}}$ is negligible because the mass splitting between two stops is small. Therefore, we here set $\phi_{A_{t}}=0$, and we scan the following four parameters,

$$
\left(\left|M_{1}\right|, \phi_{\mu}, \phi_{M_{1}}, \phi_{M_{2}}\right) \text {. }
$$

\section{OBSERVABLES}

As we mentioned in the previous section, we choose $|\mu|$ to achieve the correct DM relic density as $\Omega_{\mathrm{DM}} h^{2}=$ $0.1198 \pm 0.0015$ [44]. We use micrOMEGAs 4.3.5 [45] with CPsuperH2.3 [46] in calculations of dark matter thermal relic density and the Higgs mass. In our benchmark point, the Higgs mass is almost fixed to be $125 \mathrm{GeV}$. There is small fluctuation of order of $0.1 \mathrm{GeV}$ by scattering the parameters. On the other hand, the calculation of the Higgs mass has theoretical uncertainty of order of a few GeV. So we consider that our benchmark points are consistent with measurements of the Higgs mass at the LHC.

With the correct DM relic abundance and the correct Higgs mass, we calculate the electron EDM, the neutron
EDM, and the mercury EDM. The electron and mercury EDMs give strong constraints on the parameter space as we will see later. We also discuss the scattering cross section for the direct detection experiments.

\section{A. EDM}

The EDMs of fermions $\left(d_{f}\right)$, the EDM of electron $\left(d_{e}\right)$, the chromo EDM (cEDM) of quarks $\left(d_{q}^{C}\right)$, and the Wilson coefficient of the Weinberg operator $(\omega)$ are defined by

$$
\begin{aligned}
\mathcal{L} \supset & -d_{f} \frac{i}{2} \bar{f} \sigma^{\mu \nu} \gamma_{5} f F_{\mu \nu}-g_{s} d_{q}^{C} \frac{i}{2} \bar{q} \sigma^{\mu \nu} \gamma_{5} q G_{\mu \nu} \\
& -\omega \frac{1}{6} f^{a b c} G_{\mu \nu}^{a} G^{b \nu}{ }_{\rho} G_{\alpha \beta}^{c} \epsilon^{\rho \mu \alpha \beta},
\end{aligned}
$$

where the convention of the epsilon tensor is $\epsilon^{0123}=+1$. We calculate $d_{u}, d_{d}, d_{e}, d_{u}^{C}$, and $d_{d}^{C}$ by using CPsuperH2.3 [46] implemented in micrOMEGAs 4.3.5 [45]. We use the formulae given in Ref. [47] and couplings calculated by CPsuperH2.3 to evaluate $\omega .{ }^{3}$ These EDMs and the Wilson coefficient are evaluated at the electroweak scale $\mu_{W}=m_{t}$. The neutron EDM and the mercury EDM have to be evaluated at the hadronic scale $\left(\mu_{H} \simeq 1 \mathrm{GeV}\right)$. The renormalization group evolution from the electroweak scale to the hadronic scale is taken into account [48]. At the leading order of QCD, we find ${ }^{4}$

$$
\begin{aligned}
\frac{d_{u}}{e}\left(\mu_{H}\right)= & 0.35 \frac{d_{u}}{e}\left(\mu_{W}\right)-0.17 g_{s}\left(\mu_{W}\right) d_{u}^{C}\left(\mu_{W}\right) \\
& -\left(9.24874 \times 10^{-5} \mathrm{GeV}\right) \omega\left(\mu_{W}\right) \\
d_{u}^{C}\left(\mu_{H}\right)= & 0.34 g_{s}\left(\mu_{W}\right) d_{u}^{C}\left(\mu_{W}\right)+(0.00031 \mathrm{GeV}) \omega\left(\mu_{W}\right) \\
\frac{d_{d}^{e}}{e}\left(\mu_{H}\right)= & 0.40 \frac{d_{u}}{e}\left(\mu_{W}\right)+0.098 g_{s}\left(\mu_{W}\right) d_{d}^{C}\left(\mu_{W}\right) \\
& +(0.00010 \mathrm{GeV}) \omega\left(\mu_{W}\right) \\
d_{d}^{C}\left(\mu_{H}\right)= & 0.38 g_{s}\left(\mu_{W}\right) d_{d}^{C}\left(\mu_{W}\right)+(0.00070 \mathrm{GeV}) \omega\left(\mu_{W}\right)
\end{aligned}
$$

$\omega\left(\mu_{H}\right)=0.39 \omega\left(\mu_{W}\right)$.

Here the unit of the EDMs and of the cEDMs are $\mathrm{GeV}^{-1}$, and the unit of $\omega$ is $\mathrm{GeV}^{-2}$. In the evaluation, we used the following values,

\footnotetext{
${ }^{3}$ CPsuperH2.3 also calculate the Wilson coefficient of the Weinberg operator, but it returns very unstable numbers during the scanning the parameter space because of the loss of significant digits.

${ }^{4}$ The definition of the cEDM in the CPsuperH2.3 is different from ours, $\left.d_{q}^{C}\right|_{\text {CPSUPERH2.3 }}=g_{s} d_{q}^{C}$.
} 


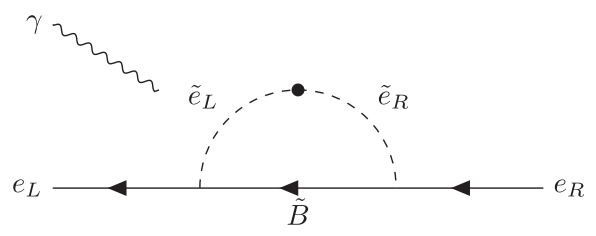

(N1)

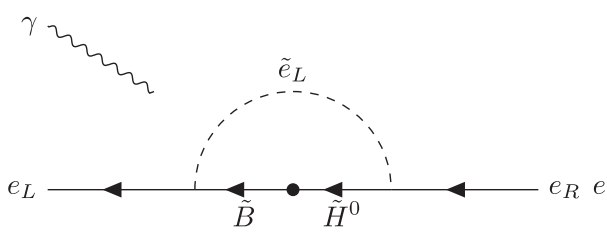

(N3)

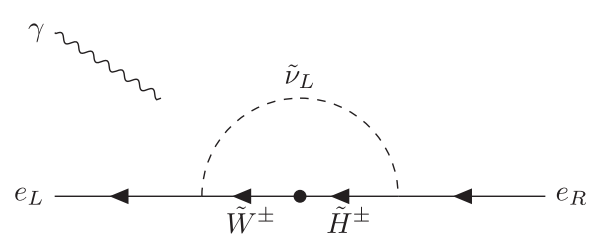

(C)

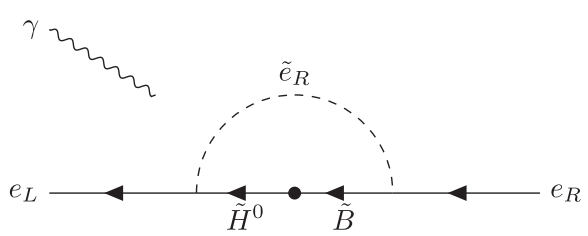

(N2)

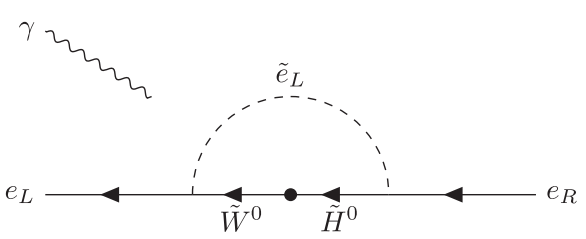

(N4)

FIG. 1. Diagrams for leading-order contributions to the electron EDM at the one-loop level.

$$
\begin{array}{r}
g_{s}\left(\mu_{W}\right)=1.1666, \quad g_{s}\left(\mu_{H}\right)=2.13309, \\
m_{u}\left(\mu_{W}\right)=0.003 \mathrm{GeV}, \quad m_{u}\left(\mu_{H}\right)=0.0024 \mathrm{GeV}, \\
m_{d}\left(\mu_{W}\right)=0.006 \mathrm{GeV}, \quad m_{d}\left(\mu_{H}\right)=0.0054 \mathrm{GeV} .
\end{array}
$$

We estimate the neutron EDM and the Mercury EDM as ${ }^{5}$

$$
\begin{aligned}
d_{n}= & 0.79 d_{d}-0.20 d_{u}+e\left(0.30 d_{u}^{C}+0.59 d_{d}^{C}\right) \\
& \pm(10-30) \mathrm{MeV} \omega, \\
\frac{d_{H g}}{e}= & 7 \times 10^{-3} \times\left(d_{u}^{C}-d_{d}^{C}\right)-10^{-2} \frac{d_{e}}{e} .
\end{aligned}
$$

Here, we used the results given in Refs. [51,52] for $d_{n}$ and the results given in Refs. $[35,36,53]$ for $d_{H g}$. We found the contribution from the Weinberg operator is much smaller than the contributions from cEDMs. Thus, we ignore the contribution from the Weinberg operator in the following analysis.

Upper bounds on the electron EDM [54], the mercury EDM [55], and the neutron EDM [56] are

$$
\left|d_{e}\right|<8.7 \times 10^{-29} \mathrm{e} \cdot \mathrm{cm} \quad(90 \% \text { C.L. }),
$$

\footnotetext{
${ }^{5}$ Some theoretical uncertainty in the EDM calculations are known. The neutron EDM calculation has uncertainty of factor two [49], and the mercury EDM calculation has 20-30\% uncertainty [50].
}

$$
\begin{gathered}
\left|d_{H g}\right|<7.4 \times 10^{-30} \mathrm{e} \cdot \mathrm{cm} \quad(95 \% \text { C.L. }) \\
\left|d_{n}\right|<2.9 \times 10^{-26} \mathrm{e} \cdot \mathrm{cm} \quad(90 \% \text { C.L. }) .
\end{gathered}
$$

We note that the constraint from the thallium EDM [57] is in practice equivalent with that to the electron EDM [58-61]. Prospects for the electron and neutron EDMs are

$$
\begin{gathered}
\left|d_{e}\right|=1 \times 10^{-30} \mathrm{e} \cdot \mathrm{cm} \quad[62,63] \\
\left|d_{n}\right|=2.5 \times 10^{-29} \mathrm{e} \cdot \mathrm{cm} \quad[64] .
\end{gathered}
$$

Let us consider the parameter dependence of the electron EDM in the MSSM. The quark EDM has the similar dependence to the electron EDM. In our setup, the EDMs are generated by the diagrams shown in Figs. 1 and 2. First, we see the one-loop contributions, where SUSY contributions are given by slepton-electroweakino loop diagrams. The relevant diagrams which give leading order contributions are shown in Fig. 1. The contribution from each diagram depends on the SUSY parameter as follows:

$$
\begin{aligned}
& d_{e}^{(\mathrm{N} 1)} \propto \operatorname{Im}\left(M_{e L R}^{2} M_{1}\right), \quad d_{e}^{(\mathrm{N} 2)} \propto \operatorname{Im}\left(\mu M_{1}\right), \\
& d_{e}^{(\mathrm{N} 3)} \propto \operatorname{Im}\left(\mu M_{1}\right), \quad d_{e}^{(\mathrm{N} 4)} \propto \operatorname{Im}\left(\mu M_{2}\right), \\
& d_{e}^{(\mathrm{C})} \propto \operatorname{Im}\left(\mu M_{2}\right) \text {, }
\end{aligned}
$$

with 


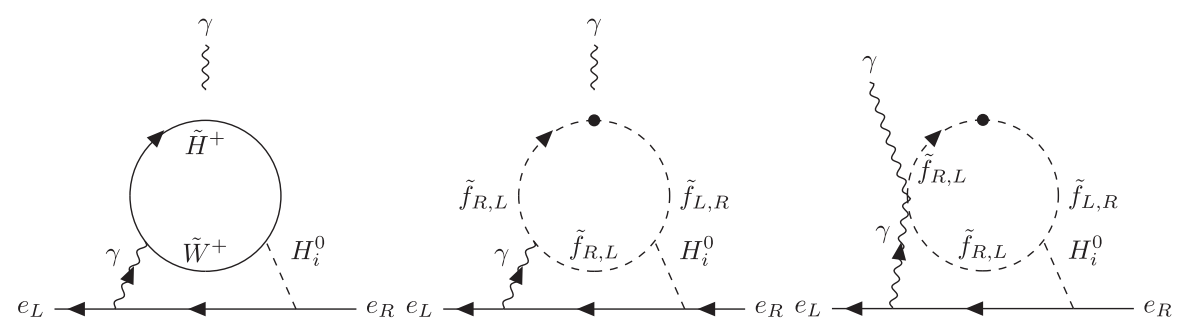

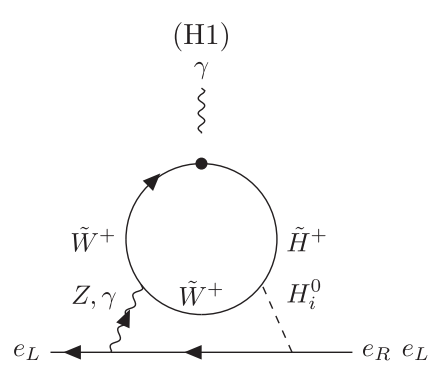

$(\mathrm{ZH})$

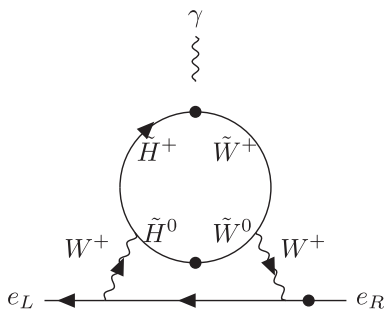

(WW)
(H2)

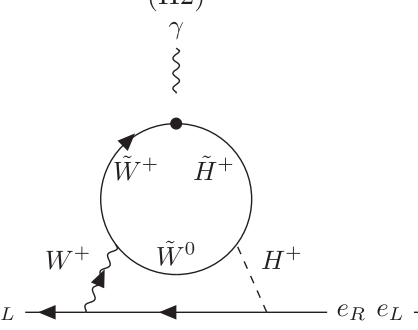

(WH1)
(H3)

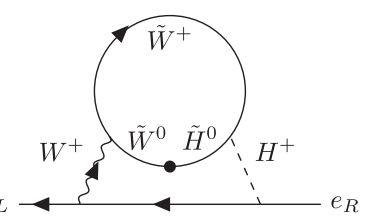

(WH2)

FIG. 2. Diagrams for leading-order contributions to the electron EDM at the two-loop level. In (H2) and (H3), stop and sbottom contributions are larger than the other sfermion contributions.

$$
M_{e L R}^{2}=A_{e}^{*} m_{e}-\mu m_{e} \tan \beta
$$

At the two-loop level, Barr-Zee diagrams shown in Fig. 2 give significant contributions. We can see that (WW) diagram is strongly suppressed compared to the other diagrams because it has not only a chirality suppression by the electron mass but also a suppression by double mass insertion in the loop. Contribution from each diagram depends on the SUSY parameter as follows:

$$
\begin{array}{ll}
d_{e}^{(\mathrm{H} 1)} \propto \operatorname{Im}\left(\mu M_{2}\right), & d_{e}^{(\mathrm{H} 2)} \propto \operatorname{Im}\left(M_{f L R}^{2 *} \mu\right), \\
d_{e}^{(\mathrm{H} 3)} \propto \operatorname{Im}\left(M_{f L R}^{2 *} \mu\right), & d_{e}^{(\mathrm{ZH})} \propto \operatorname{Im}\left(\mu M_{2}\right), \\
d_{e}^{(\mathrm{WH} 1)} \propto \operatorname{Im}\left(\mu M_{2}\right), & d_{e}^{(\mathrm{WH} 2)} \propto \operatorname{Im}\left(\mu M_{2}\right), \\
d_{e}^{(\mathrm{WW})} \propto \operatorname{Im}\left(\mu M_{2}\right), &
\end{array}
$$

with

$$
M_{f L R}^{2}= \begin{cases}A_{t}^{*} m_{t}-m_{t} \mu \cot \beta & f=t, \\ A_{f}^{*} m_{f}-m_{f} \mu \tan \beta & f=b, \tau .\end{cases}
$$

In our benchmark case, the one-loop contributions are strongly suppressed by large selectron and sneutrino masses, and the two-loop Barr-Zee diagrams provide dominant contributions unless Wino, stop, and sbottom masses are heavy enough to be decoupled. In the diagrams $(\mathrm{H} 2)$ and (H3), the stop and sbottom loops dominate the contribution, because the color factor enhances it and the Yukawa couplings are larger than the other sfermions. Therefore, in general, the $C P$ phase of $A_{t}$ gives a significant contribution to the EDMs through these two diagrams. However the contribution of $\phi_{A_{t}}$ is highly suppressed in our benchmark case, because the contributions of the stop loop to the EDMs is proportional to the $\tilde{t}_{L}-\tilde{t}_{R}$ mixing, $\theta_{\tilde{t}}$, and thus

$$
\left.d\right|_{\text {stop }} \propto \sin \theta_{\tilde{t}} \cos \theta_{\tilde{t}}\left(f\left(m_{\tilde{t}_{1}}\right)-f\left(m_{\tilde{t}_{2}}\right)\right),
$$

where $f$ is a loop function depending on the stop mass in the loop. Thanks to the relative sign of the mixing angle, the contributions from $\tilde{t}_{1}$ and $\tilde{t}_{2}$ are destructive. As a result, the EDMs are insensitive to $\phi_{A_{t}}$ for the degenerated stops masses regime. For example, the contribution to electric dipole moment $d_{e} / e$ is of the order of $10^{-30} \mathrm{~cm}$ for $\phi_{A_{t}}=30^{\circ}$, $\phi_{\mu}=\phi_{M_{1}}=\phi_{M_{2}}=0$, and $\left|M_{1}\right|=1 \mathrm{TeV}$. It is one order of magnitude below the present experimental bound, but it is within the reach of future experiment. In the diagrams (H1), $(\mathrm{ZH}),(\mathrm{WH} 1)$, and $(\mathrm{WH} 2)$, the leading order contributions 

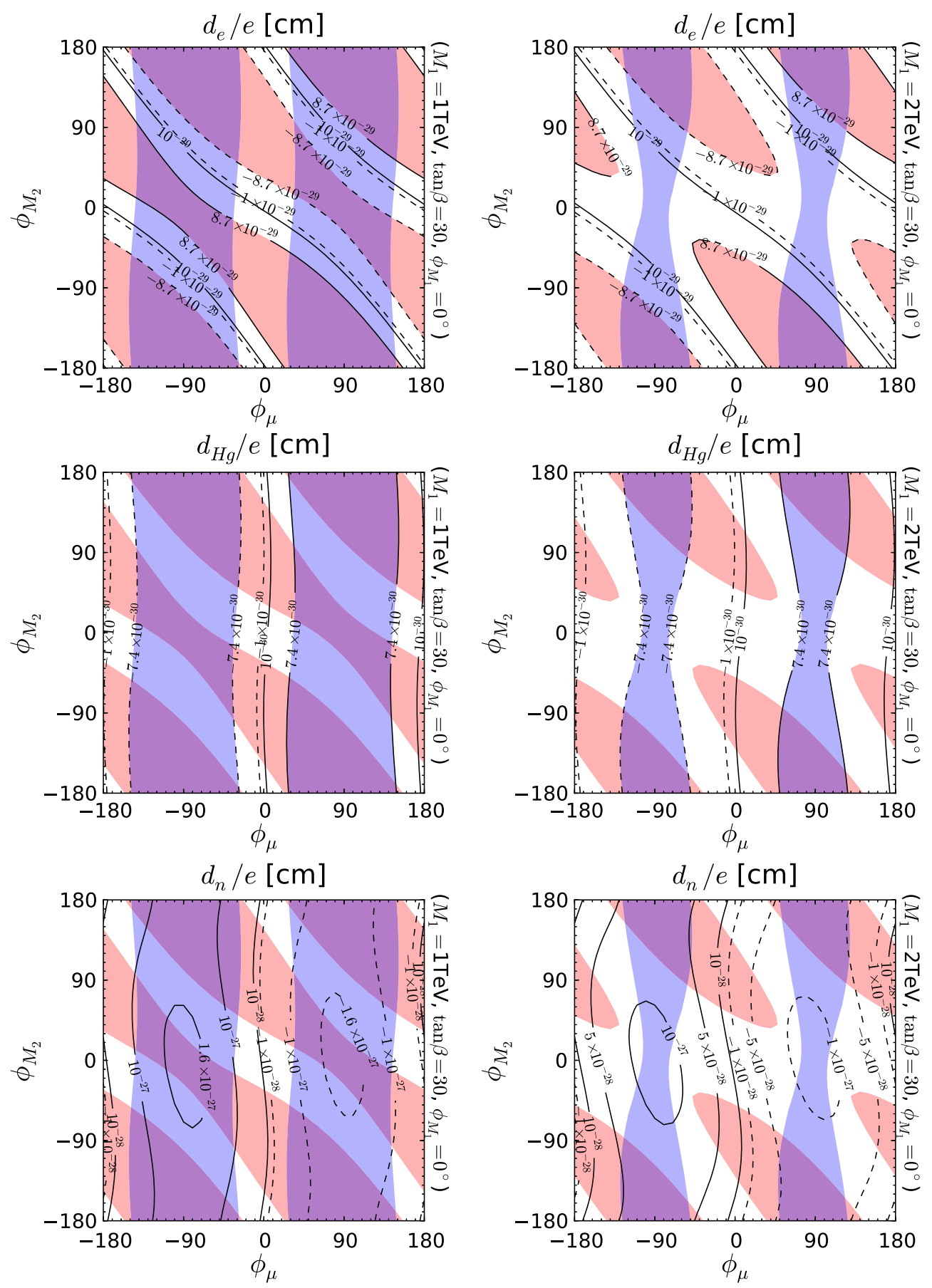

FIG. 3. The EDMs for $\tan \beta=30$ and $\phi_{M_{1}}=0^{\circ}$. The left (right) panels are for $M_{1}=1 \mathrm{TeV}\left(M_{1}=2 \mathrm{TeV}\right)$. The contours in the top, the center, and the bottom panels are those of the electron EDM, the mercury EDM, and the neutron EDM, respectively. The dashed lines show the negative values. The red and blue shaded regions are excluded by the electron EDM and the mercury EDM, respectively.

are given by the wino and the Higgsino loops so that these diagrams are decoupled when the wino mass $M_{2}$ becomes larger.

We discuss the $\phi_{\mu}$ and $\phi_{M_{2}}$ dependence of the EDMs. The left panels in Fig. 3 shows the electron EDM, the mercury EDM, and the neutron EDM with $\phi_{M_{1}}=0$. The shaded regions are already excluded by the current upper bound on the EDMs. We find the combination of the electron EDM and the mercury EDM exclude the large region of the parameter space. Both $\phi_{\mu}$ and $\phi_{M_{2}}$ cannot be large. We also find that the electron EDM strongly depends on $\phi_{M_{2}}$. On the other hand, $\phi_{M_{2}}$ dependence of the mercury EDM and the neutron EDM are milder. We focus on $M_{1}$ dependence by comparing the left panels and 
TABLE I. The mass spectrum of the SUSY particles and extra Higgs bosons in our scenario.

\begin{tabular}{|c|c|c|c|c|c|c|}
\hline Cases & $m_{\tilde{\chi}_{1}^{0}}$ & $m_{H^{ \pm}} \simeq m_{H, A}$ & $m_{\tilde{\chi}_{2,3}^{0}} \simeq m_{\tilde{\chi}_{1}^{ \pm}}$ & $m_{\tilde{\chi}_{4}^{0}} \simeq m_{\tilde{\chi}_{2}^{ \pm}} \simeq m_{\tilde{g}}$ & $m_{\tilde{t}_{1,2}} \simeq m_{\tilde{b}_{1}}$ & Other sfermions \\
\hline$M_{1}=1 \mathrm{TeV}$ & $\sim 1 \mathrm{TeV}$ & $\sim 2 \mathrm{TeV}$ & $1.6-1.7 \mathrm{TeV}$ & $\sim 10 \mathrm{TeV}$ & $\sim 7 \mathrm{TeV}$ & $\sim 100 \mathrm{TeV}$ \\
\hline$M_{1}=2 \mathrm{TeV}$ & $\sim 2 \mathrm{TeV}$ & $\sim 4 \mathrm{TeV}$ & $\sim 2.3 \mathrm{TeV}$ & $\sim 10 \mathrm{TeV}$ & $\sim 7 \mathrm{TeV}$ & $\sim 100 \mathrm{TeV}$ \\
\hline
\end{tabular}
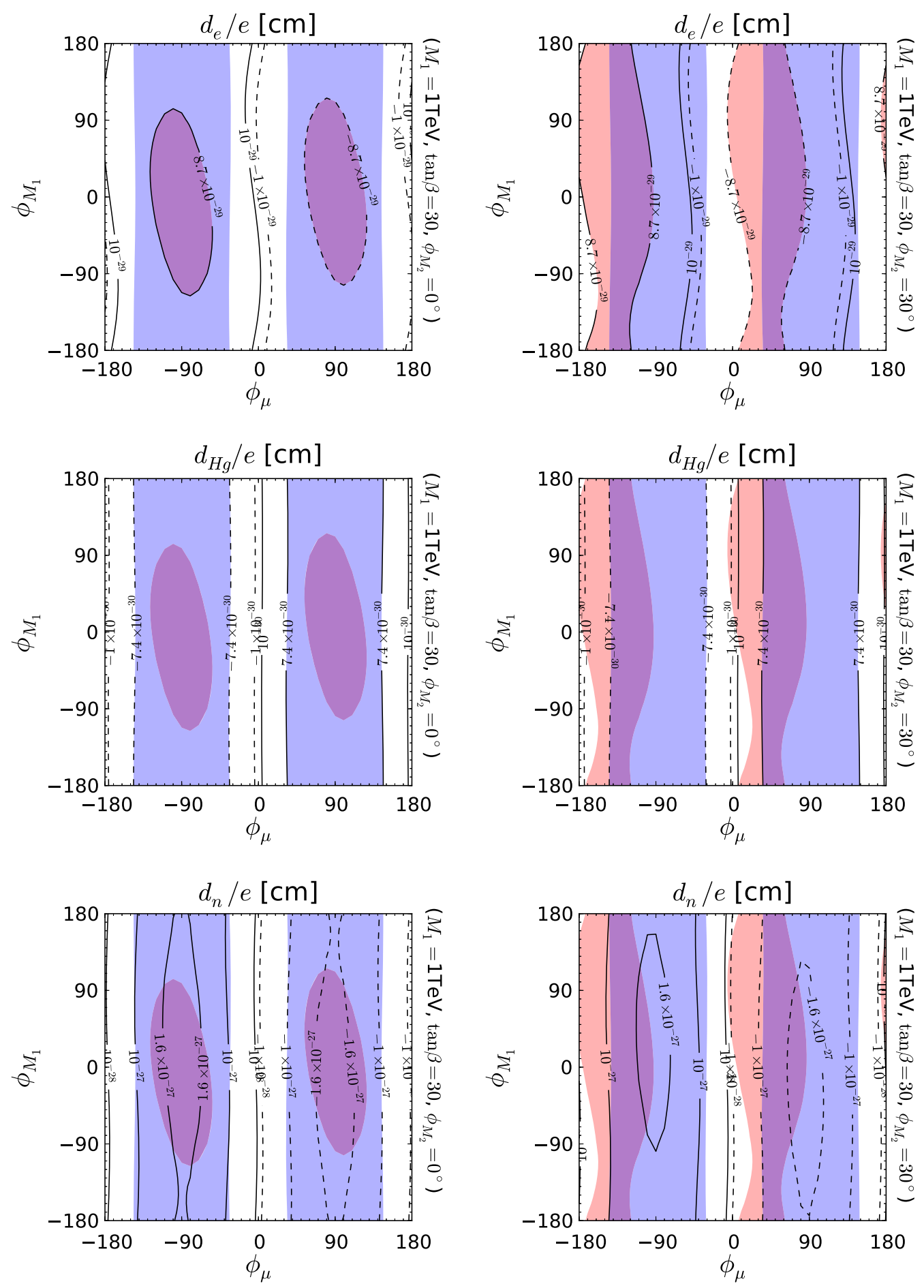

FIG. 4. The EDMs for $M_{1}=1 \mathrm{TeV}$ and $\tan \beta=30$. In the left (right) panels, $\phi_{M_{2}}=0^{\circ}\left(30^{\circ}\right)$. The shadings and contours are the same as in Fig. 3. 

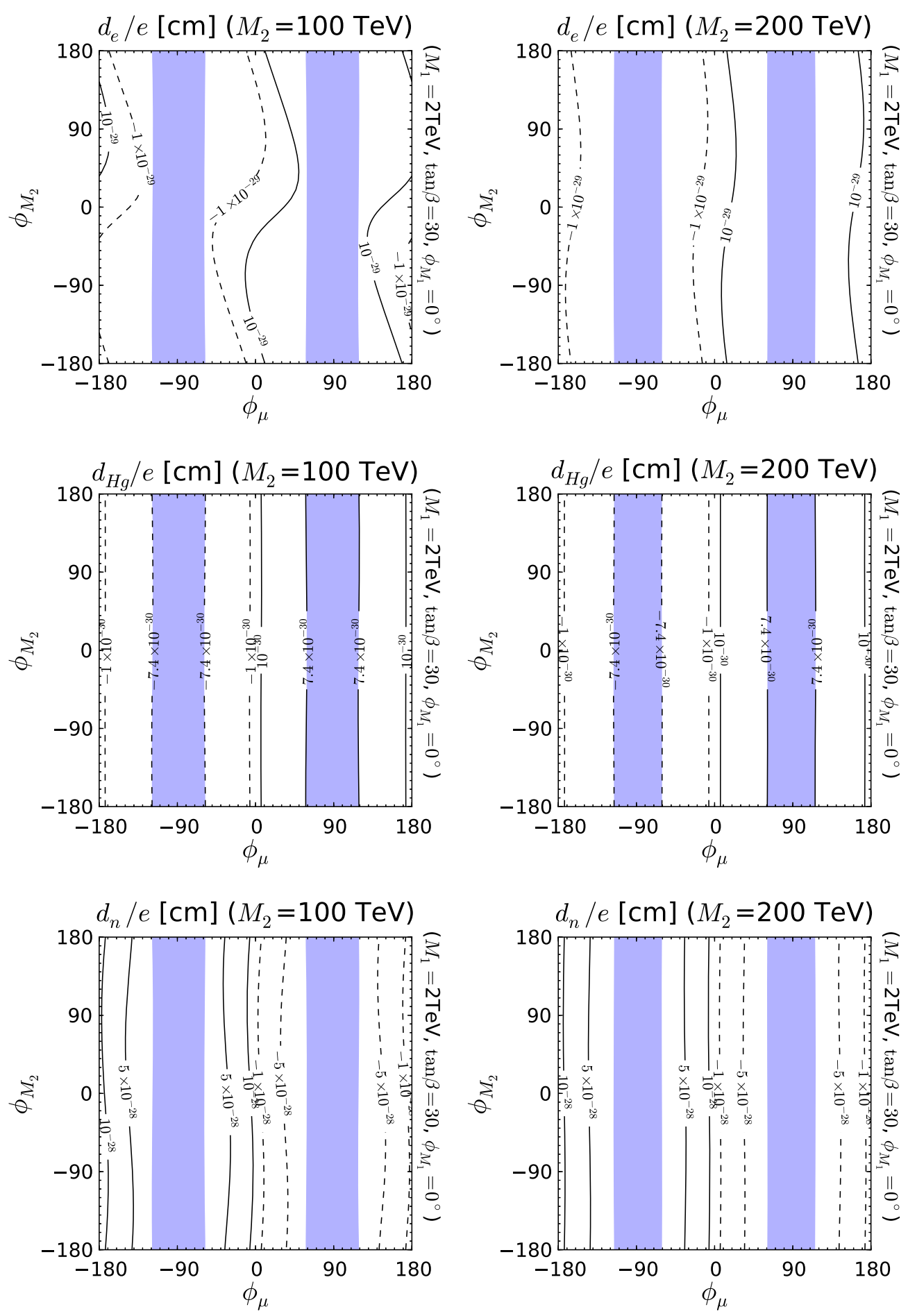

FIG. 5. The EDMs for $M_{1}=2 \mathrm{TeV}, \tan \beta=30$, and $\phi_{M_{2}}=0^{\circ}$ In the left (right) panels, $M_{2}=100 \mathrm{TeV}(200 \mathrm{TeV})$. The shadings and contours are the same as in Fig. 3. Notice that no red colored region in this parameter space.

the right panels in Fig. 3 where $M_{1}=1 \mathrm{TeV}$ and $2 \mathrm{TeV}$, respectively. We find that larger $M_{1}$ weaken the constraint from EDM experiments because $M_{1}$ is approximately the mass of the dark matter candidate here and heavy Higgs bosons and Higgsinos become heavier if we take larger $M_{1}$. Actually, the Higgsino mass $|\mu|$ is determined to be in $1.6-1.7 \mathrm{TeV}$ in order to reproduce the correct relic density of the dark matter, $\Omega_{\mathrm{DM}} h^{2}=0.1198 \pm$ 0.0015 for $M_{1}=1 \mathrm{TeV}$. For larger $M_{1}$ such as $M_{1}=2 \mathrm{TeV}$, the Higgsino mass becomes larger as $|\mu| \sim 2.3 \mathrm{TeV}$. In Table I, we show the mass spectrum of SUSY particles and the extra Higgs bosons in our benchmark points for the cases of $M_{1}=1 \mathrm{TeV}$ and $M_{1}=2 \mathrm{TeV}$. 

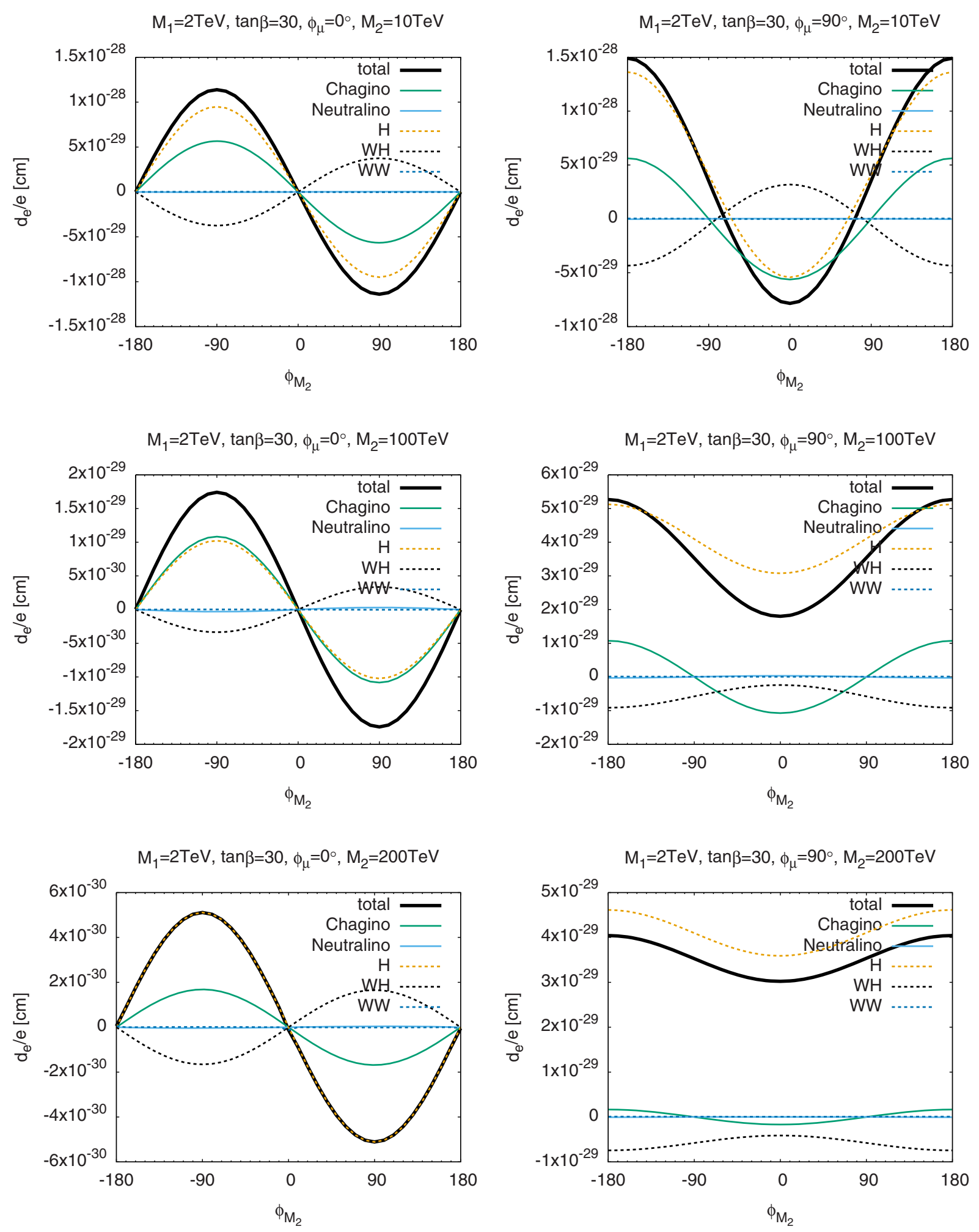

FIG. 6. Contributions to the electron EDM from each diagram. The solid green and blue curved lines are the contributions from the one-loop diagrams with chargino and neutralino, respectively. The dashed orange, dashed black, and dashed blue curved lines are the contributions from the Barr-Zee diagrams with $H-\gamma-\gamma, W^{ \pm}-H^{\mp}-\gamma$, and $W^{+}-W^{-}-\gamma$ effective vertices, respectively, where $H$ denotes three neutral Higgs bosons. 

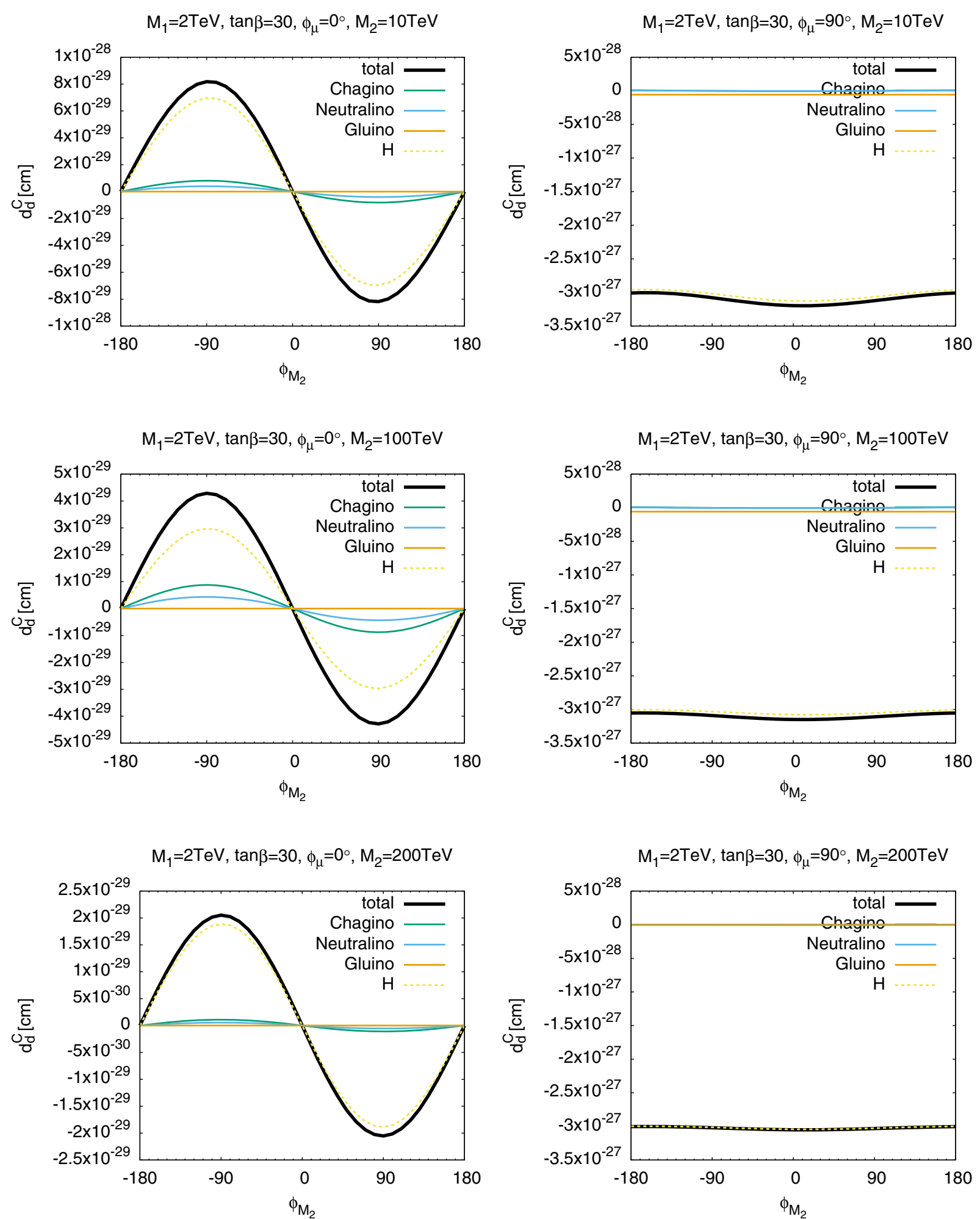

FIG. 7. Contributions to the down quark chromo-EDM from each diagram. The solid green, blue, and orange curved lines are the contributions from the one-loop diagrams with chargino, neutralino, and gluino, respectively. The dashed yellow curved lines are the contributions from the Barr-Zee diagrams with $H-g-g$.

The $\phi_{M_{1}}$ dependence can be seen in Fig. 4. After taking into account the constraint from the mercury EDM, we find that the mercury EDM and the neutron EDM are almost independent of $\phi_{M_{1}}$. On the other hand, the electron EDM has mild but visible dependence on $\phi_{M_{1}}$. Thus the electron EDM is important for determining $\phi_{M_{1}}$. 

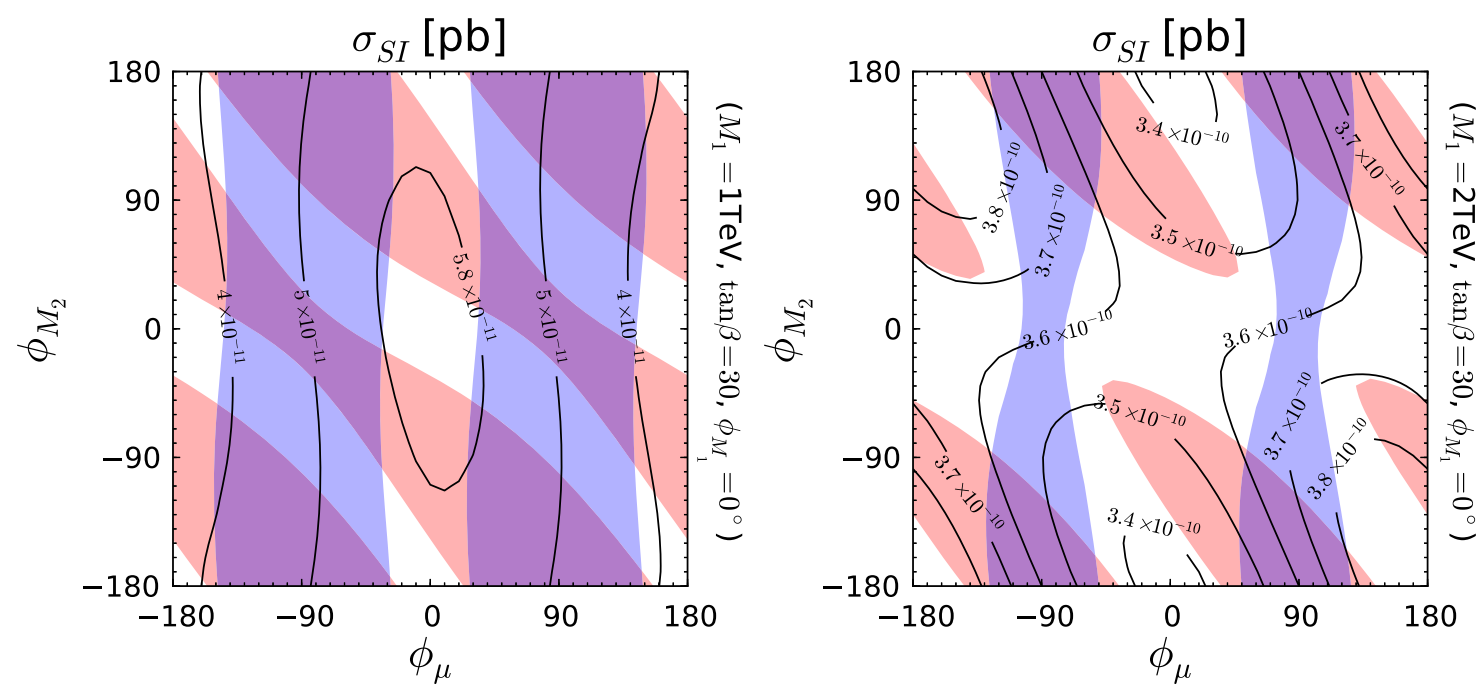

FIG. 8. The DM-nucleon scattering cross sections for $\tan \beta=30$ and $\phi_{M_{1}}=0^{\circ}$. The left (right) panel is for $\left|M_{1}\right|=1 \mathrm{TeV}(2 \mathrm{TeV})$. The shadings are the same as in Fig. 3.

Most of the parameter space in Figs. 3 and 4 are within the future prospects of the electron EDM and the neutron EDM shown in Eqs. (3.15) and (3.16). The neutron and the mercury EDMs are sensitive to $\phi_{\mu}$, and also weakly depend on $\phi_{M_{2}}$. On the other hand, the electron EDM is sensitive to $\phi_{M_{2}}+\phi_{\mu}$, and weakly depend on $\phi_{M_{1}}$. The correlation among the EDMs in future experiments provide a strong clue to explore the $C P$ violation in the SUSY breaking sector. In our scenario, we can determine the imaginary part of SUSY breaking parameters such as $\operatorname{Im}\left(M_{2}\right)$. If the absolute values of those parameters are determined by the direct measurement of SUSY particle masses, we can determine the $C P$ phases.

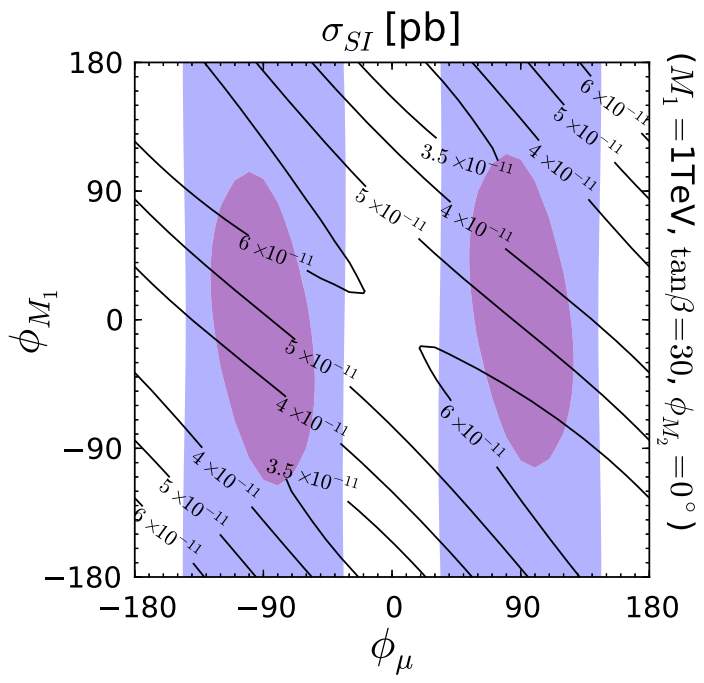

We next discuss the decoupling property for larger $M_{2}$. Figure 5 shows the EDMs for $M_{2}=100 \mathrm{TeV}$ and $200 \mathrm{TeV}$. Comparing with the right panels in Fig. 3, we find that larger $M_{2}$ drastically reduce the values of the EDMs. In particular, the electron EDM is sensitive to the $M_{2}$ choice but the mercury EDM is not. This property can be understood as follows. The dominant contribution to the electron EDM is the $H_{i}^{0}-\gamma-\gamma$ Barr-Zee diagram, where Wino loop gives the main contribution. On the other hand, the dominant contribution to the mercury EDM which originates from the quark EDMs is $H_{i}^{0}-g-g$ Barr-Zee diagram, and the diagram is independent of wino contribution. In Figs. 6 and 7, we show how each contribution to the

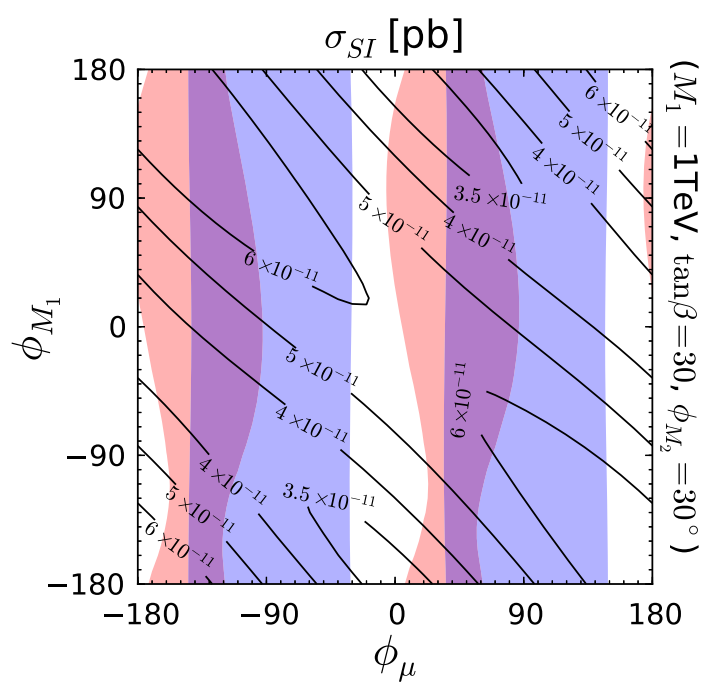

FIG. 9. The DM-nucleon scattering cross sections for $M_{1}=1 \mathrm{TeV}$ and $\tan \beta=30$. The left (right) panel is for $\left|\phi_{M_{2}}\right|=0^{\circ}\left(30^{\circ}\right)$. The shadings are the same as in Fig. 3. 
TABLE II. Prospects of sensitivity of the spin-independent cross section measurements in future experiments.

\begin{tabular}{lccc}
\hline \hline$m_{\mathrm{DM}}$ & $\mathrm{LZ}$ & DARWIN & DarkSide20k \\
\hline $1000 \mathrm{GeV}$ & $1.9 \times 10^{-11} \mathrm{pb}$ & $3.0 \times 10^{-12} \mathrm{pb}$ & $1.2 \times 10^{-11} \mathrm{pb}$ \\
$2000 \mathrm{GeV}$ & $3.7 \times 10^{-11} \mathrm{pb}$ & $5.3 \times 10^{-12} \mathrm{pb}$ & $2.3 \times 10^{-11} \mathrm{pb}$ \\
\hline \hline
\end{tabular}

electron EDM and to the down quark cEDM is decoupled for a larger value of $M_{2}$.

\section{B. DM-nucleon scattering cross section}

Since we are working in the Higgs funnel scenario, the DM candidate couples to neutral scalar bosons. The DM candidate and nucleon interact with each other through these couplings. The couplings are rather small because of the funnel scenario. However, the couplings lead to a significant size of the spin-independent cross section which is within future prospects of the DM direct detection experiments.

There is also a $Z$-exchange diagram that generates spindependent cross section. This coupling depends on the mixing between bino-Higgsino and bino-wino in the binolike DM scenario. The mixings are suppressed by the soft breaking neutralino mass parameters. We find that this coupling is so small that the resultant spin-dependent cross section $\sigma_{S D}=\mathcal{O}\left(10^{-8}\right) \mathrm{pb}$ is smaller than the prospect [65]. In the following, we focus on the spin-independent cross section.

Figure 8 shows the $\phi_{M_{2}}$ and $\phi_{\mu}$ dependence of $\sigma_{\mathrm{SI}}$ where its parameter choice is the same as in Fig. 3. Figure 9 shows the $\phi_{M_{1}}$ and $\phi_{\mu}$ dependence of $\sigma_{\mathrm{SI}}$ with the same parameter choice as in Fig. 4. We find that the spin-independent cross section is smaller than the current upper bound $[15,16,18]$ in all the region of the parameter space but within the prospects of the DARWIN [65], the DarkSide-20k [66], and the LZ [67]. We also find that the scattering cross section depends on $\phi_{M_{1}}+\phi_{\mu}$, and the $\phi_{M_{2}}$ dependence is not important. Since $\left|M_{2}\right|$ is much larger than $\left|M_{1}\right|$ and $|\mu|$ in our analysis, the sector related to dark matter physics is approximately the binoHiggsino system, and thus the scattering cross section weakly depends on $\phi_{M_{2}}$. In the bino-Higgsino system, there is only one physical $C P$ phase. This is the reason why the scattering cross section depends on one combination of the $C P$ phases, $\phi_{M_{1}}+\phi_{\mu}$.

In Table II, the future prospects of the spin-independent cross section measurements are shown. One finds that all the parameter regions in Figs. 8 and 9 are within the sensitivity of these experiments.

\section{SUMMARY}

In this paper, we have estimated EDMs of the electron, the neutron, and the mercury as well as the DM-nucleon scattering cross section and shown the present constraints and prospects in the binolike neutralino DM with the heavy Higgs funnel scenario in the $C P$-violating MSSM. In our analysis, we have fixed soft SUSY breaking parameters of stops to be $\mathcal{O}(10) \mathrm{TeV}$ and $\tan \beta=30$ to reproduce the measured SM-like Higgs boson mass and other sfermion masses to be $100 \mathrm{TeV}$ in order to be decoupled from low energy observables.

With such SUSY particle mass spectrum, we have shown that $C P$ violating phases of $\mathcal{O}(10)^{\circ}$ in the gaugino and Higgsino mass parameters are currently allowed. Future experiments will be able to constrain those phases at $\mathcal{O}(1)^{\circ}$ level if the results are null. We also pointed out that those EDMs have different phase dependence. For instance, the electron EDM mostly depends on one combination $\phi_{M_{2}}+\phi_{\mu}$, while the neutron and mercury EDMs do on mostly $\phi_{\mu}$ and weakly $\phi_{M_{2}}$. Once a few non-vanishing EDMs will be measured, it is possible to estimate individual $C P$ phases. Let us comment on the $C P$ phase of $A_{t}$. In our benchmark points with the similar size of soft stop masses and the trilinear parameter, two stop masses are relatively close so that the contribution of $\phi_{A_{t}}$ is suppressed enough for satisfying the current experimental bound. It should be noticed that even such a suppressed contribution can be tested at the future experiments. In the case of a large stop mass spliting, which is often accepted in the literature to realize the Higgs boson mass with lighter light stop mass than that we considered here, the contribution can be more significant. In such a case, we will need another observables to determine the individual $C P$ phases.

We also have calculated the dependence of spin-independent cross section of the DM in our scenario. In fact, the nonvanishing $C P$ violation effects change the cross section just by a factor. The predicted scattering cross section with a nucleon is within the sensitivity of future experiments.

Let us consider the future prospect of our scenario. We may expect a positive signal in the direct detection of the DM, which provides us an information of the DM mass. In our scenario, the extra Higgs bosons should be twice as heavy as the DM, so that the heavy Higgs search at LHC can test the scenario. If the DM mass, cross section, and the heavy Higgs masses are consistent with our scenario, we can explore the detail of SUSY breaking sector by EDM experiments even if the SUSY particles besides the DM are too heavy to be directly discovered at the future collider experiments.

\section{ACKNOWLEDGMENTS}

This work was supported by JSPS KAKENHI Grants No. 16K17715 [T. A.] and No. 17H05408 [T. S.]. This work of T.S. was also supported in part by Kogakuin University Grant for the project research. The work of N. O was supported in part by JSPS Grant-in-Aid for JSPS Fellows, No. 18J10908. 
[1] J. L. Lopez, D. V. Nanopoulos, and X. Wang, Large (g-2)$\mathrm{mu}$ in $\mathrm{SU}(5) \times \mathrm{U}(1)$ supergravity models, Phys. Rev. D 49, 366 (1994).

[2] U. Chattopadhyay and P. Nath, Probing supergravity grand unification in the Brookhaven g-2 experiment, Phys. Rev. D 53, 1648 (1996).

[3] T. Moroi, The muon anomalous magnetic dipole moment in the minimal supersymmetric standard model, Phys. Rev. D 53, 6565 (1996); Erratum, Phys. Rev. D56, 4424(E) (1997).

[4] H. Goldberg, Constraint on the Photino Mass from Cosmology, Phys. Rev. Lett. 50, 1419 (1983); Erratum, Phys. Rev. Lett.103, 099905(E) (2009).

[5] J. R. Ellis, J. S. Hagelin, D. V. Nanopoulos, K. A. Olive, and M. Srednicki, Supersymmetric relics from the big bang, Nucl. Phys. B238, 453 (1984).

[6] J. R. Ellis, S. Ferrara, and D. V. Nanopoulos, $C P$ violation and supersymmetry, Phys. Lett. 114B, 231 (1982).

[7] W. Buchmuller and D. Wyler, $C P$ violation and $\mathrm{R}$ invariance in supersymmetric models of strong and electroweak interactions, Phys. Lett. 121B, 321 (1983).

[8] J. Polchinski and M. B. Wise, The electric dipole moment of the neutron in low-energy supergravity, Phys. Lett. 125B, 393 (1983).

[9] M. Dugan, B. Grinstein, and L. J. Hall, $C P$ violation in the minimal $N=1$ supergravity theory, Nucl. Phys. B255, 413 (1985).

[10] P. Nath, $C P$ Violation via Electroweak Gauginos and the Electric Dipole Moment of the Electron, Phys. Rev. Lett. 66, 2565 (1991).

[11] Y. Kizukuri and N. Oshimo, Implications of the neutron electric dipole moment for supersymmetric models, Phys. Rev. D 45, 1806 (1992).

[12] A. Arbey, J. Ellis, R. M. Godbole, and F. Mahmoudi, Constraints on the $C P$-Violating MSSM, Nucl. Part. Phys. Proc. 285-286, 160 (2017).

[13] J. Berger, M. W. Cahill-Rowley, D. Ghosh, J. L. Hewett, A. Ismail, and T. G. Rizzo, $C P$-violating phenomenological MSSM, Phys. Rev. D 93, 035017 (2016).

[14] D. S. Akerib et al. (LUX Collaboration), First Results from the LUX Dark Matter Experiment at the Sanford Underground Research Facility, Phys. Rev. Lett. 112, 091303 (2014).

[15] D. S. Akerib et al. (LUX Collaboration), Results from a Search for Dark Matter in the Complete LUX Exposure, Phys. Rev. Lett. 118, 021303 (2017).

[16] E. Aprile et al. (XENON Collaboration), First Dark Matter Search Results from the XENON1T Experiment, Phys. Rev. Lett. 119, 181301 (2017).

[17] A. Tan et al. (PandaX-II Collaboration), Dark Matter Results from First 98.7 Days of Data from the PandaX-II Experiment, Phys. Rev. Lett. 117, 121303 (2016).

[18] X. Cui et al. (PandaX-II Collaboration), Dark Matter Results From 54-Ton-Day Exposure of PandaX-II Experiment, Phys. Rev. Lett. 119, 181302 (2017).

[19] N. Nagata and S. Shirai, Higgsino dark matter in high-scale supersymmetry, J. High Energy Phys. 01 (2015) 029.

[20] M. Drees and M. M. Nojiri, The neutralino relic density in minimal $N=1$ supergravity, Phys. Rev. D 47, 376 (1993).
[21] H. Baer and M. Brhlik, Cosmological relic density from minimal supergravity with implications for collider physics, Phys. Rev. D 53, 597 (1996).

[22] H. Baer and M. Brhlik, Neutralino dark matter in minimal supergravity: Direct detection versus collider searches, Phys. Rev. D 57, 567 (1998).

[23] V. D. Barger and C. Kao, Relic density of neutralino dark matter in supergravity models, Phys. Rev. D 57, 3131 (1998).

[24] J. R. Ellis, T. Falk, G. Ganis, K. A. Olive, and M. Srednicki, The CMSSM parameter space at large tan beta, Phys. Lett. B 510, 236 (2001).

[25] G. Belanger, O. Kittel, S. Kraml, H.-U. Martyn, and A. Pukhov, Neutralino relic density from ILC measurements in the CPV MSSM, Phys. Rev. D 78, 015011 (2008).

[26] G. Jungman, M. Kamionkowski, and K. Griest, Supersymmetric dark matter, Phys. Rep. 267, 195 (1996).

[27] P. Draper and H. Rzehak, A review of Higgs mass calculations in supersymmetric models, Phys. Rep. 619, 1 (2016).

[28] B. C. Allanach and A. Voigt, Uncertainties in the lightest $C P$ even Higgs boson mass prediction in the minimal supersymmetric standard model: Fixed order versus effective field theory prediction, Eur. Phys. J. C 78, 573 (2018).

[29] T. Falk, K. A. Olive, and M. Srednicki, Phases in the MSSM, electric dipole moments and cosmological dark matter, Phys. Lett. B 354, 99 (1995).

[30] P. Gondolo and K. Freese, $C P$ violating effects in neutralino scattering and annihilation, J. High Energy Phys. 07 (2002) 052.

[31] M. E. Gomez, T. Ibrahim, P. Nath, and S. Skadhauge, Sensitivity of supersymmetric dark matter to the $b$ quark mass, Phys. Rev. D 70, 035014 (2004).

[32] T. Nihei and M. Sasagawa, Relic density and elastic scattering cross-sections of the neutralino in the MSSM with $C P$ violating phases, Phys. Rev. D 70, 055011 (2004); Erratum, Phys. Rev. D70, 079901(E) (2004).

[33] S. Y. Choi and Y. G. Kim, Heavy Higgs resonances for the neutralino relic density in the Higgs decoupling limit of the $C P$-noninvariant minimal supersymmetric standard model, Phys. Lett. B 637, 27 (2006).

[34] G. Belanger, F. Boudjema, S. Kraml, A. Pukhov, and A. Semenov, Relic density of neutralino dark matter in the MSSM with $C P$ violation, Phys. Rev. D 73, 115007 (2006).

[35] D. A. Demir, O. Lebedev, K. A. Olive, M. Pospelov, and A. Ritz, Electric dipole moments in the MSSM at large tan beta, Nucl. Phys. B680, 339 (2004).

[36] K. A. Olive, M. Pospelov, A. Ritz, and Y. Santoso, $C P$-odd phase correlations and electric dipole moments, Phys. Rev. D 72, 075001 (2005).

[37] U. Chattopadhyay, T. Ibrahim, and P. Nath, Effects of $C P$ violation on event rates in the direct detection of dark matter, Phys. Rev. D 60, 063505 (1999).

[38] T. Falk, A. Ferstl, and K. A. Olive, New contributions to neutralino elastic cross-sections from $C P$ violating phases in the MSSM, Phys. Rev. D 59, 055009 (1999); Erratum, Phys. Rev. D60, 119904(E) (1999).

[39] T. Falk, A. Ferstl, and K. A. Olive, Variations of the neutralino elastic cross-section with $C P$ violating phases, Astropart. Phys. 13, 301 (2000). 
[40] S. Y. Choi, S. C. Park, J. H. Jang, and H. S. Song, Neutralino nucleus elastic cross-section in the minimal supersymmetric standard model with explicit $C P$ violation, Phys. Rev. D 64 , 015006 (2001).

[41] T. Abe, R. Kitano, and R. Sato, Discrimination of dark matter models in future experiments, Phys. Rev. D 91, 095004 (2015); Erratum, Phys. Rev. D96, 019902(E) (2017).

[42] T. Abe, Effect of $C P$ violation in the singlet-doublet dark matter model, Phys. Lett. B 771, 125 (2017).

[43] B. C. Allanach et al., SUSY Les Houches accord 2, Comput. Phys. Commun. 180, 8 (2009).

[44] P. A. R. Ade et al. (Planck Collaboration), Planck 2015 results. XIII. Cosmological parameters, Astron. Astrophys. 594, A13 (2016).

[45] D. Barducci, G. Belanger, J. Bernon, F. Boudjema, J. Da Silva, S. Kraml, U. Laa, and A. Pukhov, Collider limits on new physics within micrOMEGAs_4.3, Comput. Phys. Commun. 222, 327 (2018).

[46] J. S. Lee, M. Carena, J. Ellis, A. Pilaftsis, and C. E. M. Wagner, CPSUPERH2.3: An updated tool for phenomenology in the MSSM with explicit $C P$ violation, Comput. Phys. Commun. 184, 1220 (2013).

[47] T. Abe, J. Hisano, and R. Nagai, Model independent evaluation of the Wilson coefficient of the Weinberg operator in QCD, J. High Energy Phys. 03 (2018) 175.

[48] G. Degrassi, E. Franco, S. Marchetti, and L. Silvestrini, QCD corrections to the electric dipole moment of the neutron in the MSSM, J. High Energy Phys. 11 (2005) 044.

[49] J. Hisano, Review of electric dipole moments (EDMs) in Proceedings, 49th Rencontres de Moriond on Electroweak Interactions and Unified Theories: La Thuile, Italy, 2014 (ARISF, Paris, 2014), pp. 285-292.

[50] J. S. M. Ginges and V. V. Flambaum, Violations of fundamental symmetries in atoms and tests of unification theories of elementary particles, Phys. Rep. 397, 63 (2004).

[51] D. A. Demir, M. Pospelov, and A. Ritz, Hadronic EDMs, the Weinberg operator, and light gluinos, Phys. Rev. D 67, 015007 (2003).

[52] K. Fuyuto, J. Hisano, N. Nagata, and K. Tsumura, QCD corrections to quark (chromo)electric dipole moments in high-scale supersymmetry, J. High Energy Phys. 12 (2013) 010 .
[53] J. R. Ellis, J. S. Lee, and A. Pilaftsis, Electric dipole moments in the MSSM reloaded, J. High Energy Phys. 10 (2008) 049.

[54] J. Baron et al. (ACME Collaboration), Order of magnitude smaller limit on the electric dipole moment of the electron, Science 343, 269 (2014).

[55] B. Graner, Y. Chen, E. G. Lindahl, and B. R. Heckel, Reduced Limit on the Permanent Electric Dipole Moment of Hg199, Phys. Rev. Lett. 116, 161601 (2016); Erratum, Phys. Rev. Lett.119, 119901(E) (2017).

[56] C. A. Baker et al., An Improved Experimental Limit on the Electric Dipole Moment of the Neutron, Phys. Rev. Lett. 97, 131801 (2006).

[57] B. C. Regan, E. D. Commins, C. J. Schmidt, and D. DeMille, New Limit on the Electron Electric Dipole Moment, Phys. Rev. Lett. 88, 071805 (2002).

[58] Z. W. Liu and H. P. Kelly, Collider limits on new physics within micrOMEGAs_4.3, Phys. Rev. A 45, R4210 (1992).

[59] A.-M. Martensson-Pendrill, Methods in Computational Chemistry, edited by S. Wilson, Atomic, Molecular Properties (Plenum Press, New York, 1992), Vol. 5.

[60] E. Lindroth and A.-M. Martensson-Pendrill, Limit on a P- and T-violating electron-nucleon interaction, Europhys. Lett. 15, 155 (1991).

[61] M. Pospelov and A. Ritz, Electric dipole moments as probes of new physics, Ann. Phys. (Amsterdam) 318, 119 (2005).

[62] D. M. Kara, I. J. Smallman, J. J. Hudson, B. E. Sauer, M. R. Tarbutt, and E. A. Hinds, Measurement of the electron's electric dipole moment using YbF molecules: Methods and data analysis, New J. Phys. 14, 103051 (2012).

[63] D. Kawall, Searching for the electron EDM in a storage ring, J. Phys. Conf. Ser. 295, 012031 (2011).

[64] A. Lehrach, B. Lorentz, W. Morse, N. Nikolaev, and F. Rathmann, Precursor experiments to search for permanent electric dipole moments (EDMs) of protons and deuterons at COSY, arXiv:1201.5773.

[65] J. Aalbers et al. (DARWIN Collaboration), DARWIN: Towards the ultimate dark matter detector, J. Cosmol. Astropart. Phys. 11 (2016) 017.

[66] C. E. Aalseth et al., DarkSide-20k: A 20 tonne two-phase LAr TPC for direct dark matter detection at LNGS, Eur. Phys. J. Plus 133, 131 (2018).

[67] D. S. Akerib et al. (LUX-ZEPLIN Collaboration), Projected WIMP sensitivity of the LUX-ZEPLIN (LZ) dark matter experiment, arXiv:1802.06039. 